\title{
Arthroscopic Primary Repair of the Anterior Cruciate Ligament: A Literature Review
}

\author{
Salim Al Rawahi ${ }^{1}$, Humaid Al Farii ${ }^{1,}$, , Sultan Al Maskari ${ }^{2}$ \\ ${ }^{1}$ Division of Orthopaedic Surgery, McGill University, Montreal, Qc, Canada \\ ${ }^{2}$ Division of Orthopaedic Surgery, Sultan Qaboos University, Muscat, Oman
}

Email address:

Humaid44@gmail.com (H. Al Farii)

${ }^{*}$ Corresponding author

\section{To cite this article:}

Salim Al Rawahi, Humaid Al Farii, Sultan Al Maskari. Arthroscopic Primary Repair of the Anterior Cruciate Ligament: A Literature Review. American Journal of Sports Science. Vol. 8, No. 3, 2020, pp. 68-72. doi: 10.11648/j.ajss.20200803.14

Received: July 4, 2020; Accepted: July 21, 2020; Published: August 17, 2020

\begin{abstract}
Introduction: Anterior cruciate ligament (ACL) is an important mechanical stabilizer of the knee joint and ACL injuries are commonly seen among athletes. The gold-standard treatment adopted worldwide for ACL injuries is arthroscopic ACL reconstruction. However, arthroscopic primary ACL repair has gained popularity recently. The aim of this review is to provide an overall update of the studies that looked at arthroscopic primary ACL repair. Methods: Electronic databases were searched for relevant studies linked to arthroscopic primary ACL repair. We excluded any nonsurgical treatment studies, cadaveric studies and review articles. Nine hundred ninety-five articles were identified for screening, and a total of 523 patients from 8 articles were included for the review. Results: The study included articles published between 1985 and 2019: $66.6 \%$ were male, median age was 27 years; and $97.7 \%$ had sport related injuries. Two-hundred and nineteen patients had acute arthroscopic primary repair with varying types of ACL fixation; 287 of them had concomitant injuries. Rehabilitation program varies from a study to another; average evaluation for return to sport was 6 months. Eleven cases of the primary ACL repair have failed. Overall, patients reported satisfactory to good results. Conclusion: Arthroscopic primary ACL repair is a promising procedure with advantages over ACL reconstruction. However, appropriate patients' selection, surgical expertise and instrumentations, pre- and post-op rehabilitation programs are all contributing factors to successful outcomes.
\end{abstract}

Keywords: Anterior Cruciate Ligament, Arthroscopy, Repair

\section{Introduction}

Anterior cruciate ligament (ACL) is an important mechanical stabilizer during knee-joint movement; it is the primary restraint against anterior tibial translation relative to the femur; and it acts as secondary restraint to tibial rotation and varus/valgus rotation. ACL injuries are commonly seen among athletics; and the number is increasing, with a reported incidence of $38 / 100,000$ per year $[1,2]$. Although there is still some debate about the optimal treatment for acute ACL tears, surgical management in the young and active patient is recommended [3-6]. The surgical management options to an isolated ACL tear vary from acute repair to ligament reconstruction. Several studies demonstrated that the results of acute repair of ACL ruptures deteriorate with time and the procedure was shown to be ineffective [7-10]. However, most of the published studies are retrospective and nonrandomized, and they compare different surgical techniques, rehabilitation, and follow-up protocols.

There is a dearth of studies looking into the outcomes of arthroscopic primary ACL repair worldwide, and therefore, the current literature review aims to provide an overall update of these studies.

\section{Methods}

\subsection{Search Strategy}

The research question and inclusion/exclusion criteria for individual studies were established prior to searching databases. Online databases (PubMed and Medline) were used to find literature related to arthroscopic primary repair 
of the ACL. Key words included were "anterior cruciate ligament" and "Arthroscopy" and "Repair". Searches was conducted on October $1^{\text {st }} 2019$ and yielded a total of nine hundred and ninety-five articles from both databases, without any restriction on language or date of publication (Table 1).

Table 1. Search strategy of PubMed and MEDLINE databases.

\begin{tabular}{ll}
\hline Medline October $\mathbf{1}^{\text {st }} \mathbf{2 0 1 9}$ & Total articles \\
\hline 1) "Anterior cruciate ligament" & 20172 \\
2) “Arthroscopy" & 27696 \\
3) "Repair" & 311650 \\
4) 1, 2 and 3 & 496 \\
PubMed October 1 $1^{\text {st }} 2019$ & \\
1) “Anterior cruciate ligament" & 21455 \\
2) "Arthroscopy" & 32220 \\
3) "Repair" & 472817 \\
4) 1, 2 and 3 & 499 \\
Total from PubMed and Medline & 995 \\
\hline
\end{tabular}

The following inclusion criteria were used: (1) all levels of evidence, (2) male and female patients with no age limitation, (4) studies on humans, (5) ACL rupture from its femoral attachment or a proximal one third tear. We excluded articles including patients with mid-substance ACL tear or who have previous surgery in the injured knee or associated knee pathologies (e.g. infection; fractures, osteoarthritis, etc.). We also excluded any nonsurgical treatment studies (e.g. conservative treatment), cadaveric studies and review articles.

\subsection{Study Selection}

Combined results of all searches produced thirty-eight articles. In the first reviewing stage, titles and abstracts were screened, as well as titles and abstracts of crossover references. Eight studies satisfied all inclusion and exclusion criteria. An independent reviewer performed a full-text review of the eight eligible studies (Figure 1).

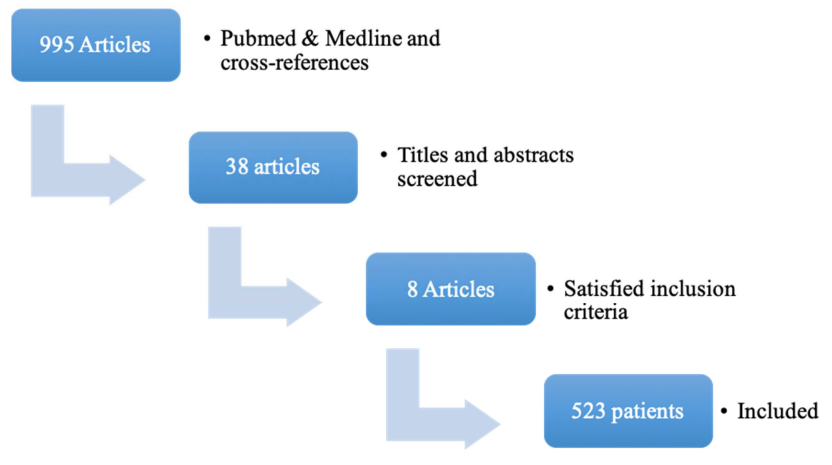

Figure 1. Flow chart illustrating the article screening process.

\subsection{Data Abstraction}

One reviewer abstracted relevant study data from the final pool of included studies and recorded them on a spreadsheet. These included study and publication information (author, year of publication, study design, level of evidence, and sample size), patients' data (age, sex, and mechanism of injury), surgical details (time to surgery, type of ACL fixation performed, and complications), post-operative rehabilitation program, assessment tools and scores used pre- and postoperatively to assess the outcomes, failure rate, follow-up, and limitations of all studies included.

\section{Results}

The articles included were published between 1985 and 2019 (Table 2). One of the studies was a prospective cohort while the rest were retrospective. The cumulative sample size was 523 patients. The age of the patients ranges from 13 to 67 years with a median age of 27 years. Male gender predominance accounts for $66.6 \%$. Nearly $97.7 \%$ of the patients had sport-related injuries in seven studies; one of the studies did not mention the mechanism of injury.

Table 2. Studies included for review. NR (Not Reported).

\begin{tabular}{|c|c|c|c|c|c|c|c|}
\hline Study & $\begin{array}{l}\text { Level of } \\
\text { evidence }\end{array}$ & $\begin{array}{l}\text { Sample } \\
\text { size }\end{array}$ & $\begin{array}{l}\text { Age "Year" } \\
\text { (Mean/Median) }\end{array}$ & $\begin{array}{l}\text { Male Sex } \\
(\%)\end{array}$ & $\begin{array}{l}\text { Follow up } \\
\text { "Months" }\end{array}$ & Technique & $\begin{array}{l}\text { Failure of } \\
\text { primary ACL } \\
\text { repair (n) }\end{array}$ \\
\hline Andersson, et al. 1989 [23] & II & 71 & $25(+/-6)$ & $76 \%$ & 48 & $\begin{array}{l}\text { Primary repair: NR } \\
\text { Augmented repair: Iliotibial strip }\end{array}$ & NR \\
\hline Fox JM, et al. 1985 [30] & II & 5 & 35.2 & $60 \%$ & 10 & Non-absorbable sutures & NR \\
\hline DiFelice, et al. 2015 [27] & IV & 11 & 37 & $90.9 \%$ & 24 & Suture anchors & 1 \\
\hline Achtnich, et al. 2016 [26] & III & 41 & $\begin{array}{l}\text { Repair group: } 30(+/-8.9) \\
\text { Control group: } 33.6(+/-3.7)\end{array}$ & NR & 31 & Suture anchors & 3 \\
\hline DiFelice, et al. 2018 [28] & IV & 10 & 37 & $90 \%$ & 60 & Suture anchors & 0 \\
\hline Hoffmann, et al. 2017 [29] & III & 13 & 43.3 & $30.8 \%$ & 98 & Suture anchors & 3 \\
\hline Van der List, et al 2019 [24] & III & 316 & 28 & $59.8 \%$ & 120 & NR & NR \\
\hline Jonkergouw, et al. 2019 [25] & III & 56 & $33.5(+/-11.3)$ & $58.9 \%$ & 38.4 & Suture anchors & 4 \\
\hline
\end{tabular}

ACL tear was assessed clinically by manual laxity tests (anterior drawer test, Lachman's test, and pivot shift test) and was confirmed by knee MRI. Knee X-rays were also done to rule out associated knee pathologies. Out of 524, 379 patients $(72.3 \%)$ were assessed clinically and by MRI while 5 patients were assessed clinically and under anesthesia before and during arthroscopy. In two studies; the mode of assessment was not documented clearly.

For 71 patients who underwent arthroscopic ACL repair (61 augmented vs. 10 non-augmented), time from injury to surgery was not documented. Only 219 patients (48.3\%) had their ACL repaired acutely within three months while 29 patients underwent arthroscopic ACL primary repair within a median of 203 days. 
Type of ACL fixation varied between the studies; five studies used FiberWires sutures and a single PushLock anchor; one study used non-absorbable sutures alone; and 1 study used an augmented repair with an Iliotibial strip for one group. However, the later study did not document the primary ACL repair technique for the second group. Type of ACL fixation was not mentioned in one of the included studies. The follow up time after the surgery ranges from eight months to ten years.

Fifty-two patients with ACL injuries had a concomitant medial collateral ligament (MCL) injury, 6 patients had a lateral collateral ligament (LCL) injury, 84 patients had a lateral meniscus (LM) injury, 77 patients had a medial meniscus (MM) injury, 66 patients had a chondral lesion, 1 patient had a patellar tendon rupture and 1 patient had a chondromalacia. Only 3 patients underwent LCL femoral avulsion screw fixation, MCL tibial avulsion screw fixation, and patella tendon reconstruction simultaneously with the arthroscopic primary ACL repair.

Post-operative rehabilitation program differs from a study to another, however, all patients in each study followed the same protocol regardless of the ACL fixation type. Twelve patients were allowed partial weight bearing for two weeks, to wear a knee brace in full extension, with flexion limitation to 90 degrees for six weeks; after the six weeks, patients follow standard ACL rehabilitation protocol. For 71 patients, a knee immobilizer in a cast was placed for five weeks, during which, weight-bearing was not allowed; this was followed by a graduated exercise program and continued until the patient regained at least $85 \%$ of normal muscle strength. Five patients were instructed to wear a compression dressing plus anterior and posterior splints in $30^{\circ}$ of flexion for three weeks and then bracing, allowing motion between 30-90 degrees for three weeks; weight bearing status was not documented in this study. Weight bearing was allowed for 56 patients with the brace locked in extension, until quadriceps muscle control had been regained (examined at approximately four weeks post-operatively); formal physical therapy for these patients was started after four weeks. Fortyone patients wore a knee brace for two weeks to maintain the leg in full extension, then the brace settings were changed to allow 90 degrees of knee flexion for the following four weeks; partial weight bearing was permitted for six weeks. For twenty-one patients, a brace was worn for the first month, with weight bearing as tolerated. Range-of-motion exercises were initiated in the first few days after surgery in a controlled fashion. The brace was weaned off and formal therapy was started after the first month. At four to six weeks postoperatively, the patient was advanced to gentle strengthening and placed on a standard ACL rehabilitation protocol. For 316 patients, no rehabilitation protocol was documented in the study.

It was not documented when patients with acute primary ACL repair went back to sport and high activity levels. However, at six months' evaluation post-operatively, patients were assessed for readiness to go back to sport. Two patients, who underwent acute primary ACL repair, returned to full contact sports on their own volition within 4 months postoperatively, and had excellent objective and subjective outcomes.

Different scores were used to evaluate the outcomes, e.g., Modified Cincinnati score, Tegner activity score (pre- and post-operative), Lysholm score, Subjective International Knee Documentation Committee (IKDC) score, Single Assessment Numeric Evaluation (SANE), KT-1000 Knee Ligament Arthrometer, and UCLA Instrumented Clinical Knee Testing Apparatus.

There were no immediate post-operative complications observed. Two patients lost to follow-up after the surgery; and 11 patients failed arthroscopic ACL primary repair.

\section{Discussion}

Acute arthroscopic ACL repair can be classified into: acute primary repair, acute repair with the use of a ligament augmentation device, and acute repair with augmentation with an autologous tendon graft.

The concept of ACL potential healing has been deserted in the past, however, there is renewed interest in the primary healing capacity of this ligament $[11,12]$. One may argue why is there a need for the repair of a torn ACL. First, the operation is simple and takes less time and less risk in comparison to ACL reconstruction. Second, ACL treatment aims to provide immediate knee stability; resolve the pain and avoiding long-term complications, primarily osteoarthritis. ACL reconstruction using an autologous or allogenic tendon graft, is universally accepted as the gold standard treatment for complete ACL tears, as it yields excellent results for instability and pain in most the patients. However, there is a higher failure rate in adolescents than in other age groups with up to $20 \%$ to $25 \%$ of patients experiencing problems postoperatively [13]. This mandates to find a better solution for this group of patients. In addition, patients post ACL reconstructions were found to have higher rates of osteoarthritis, despite the treatment; these findings are based on long-term follow up studies [14-17]. This emphasizes on the need to find an alternative surgical management option to improve the ultimate outcomes. Third, the incidence of ACL injuries in skeletally immature patients are increasing; and so far, there are no standardized treatment options [13]. For this group of patients, the transphyseal ACL reconstruction with grafts carries the risk of growth plate arrest $[18,19]$. although several studies have proven no increase in the rate of such complications [20-22]. Hence, the pre-pubescent population has the potential to benefit greatly from this reparative treatment, which does not violate the physes; and they respond remarkably well to certain biological stimuli implemented in the current enhanced repair techniques $[11,12]$. Fourth, repair of the ACL preserves the remaining proprioceptive fibers within the ligament substance, and maintains the complex insertion sites of the ligament [22]. This could potentially lead to more normal biomechanics of the knee if adequate repair is achieved [22].

In our study, different types of fixation were used; failure 
rate was not documented for repair using Iliotibial strip. However, most of the studies have used suture anchor with favorable results. Majority of the patients who underwent acute primary ACL repair had concomitant injuries, which were not addressed concurrently with ACL repair, except for 3 patients. In one of the studies, patients with ACL tear were categorized into 3 categories as grade I, II, and III; few of the patients had chondral lesions and meniscal tears which were resected, repaired or left untreated. The study showed no difference in activity level between grades I, II, and III and no difference in the functional outcome between patients with superficial and localized lesions and those with no chondral lesions. However, patients with grade III injuries had slightly less functional outcome compared to patients with grade I and II. The study showed also that patients with augmented repair had a higher functional score than those with primary repair alone [23]. It was concluded in one of the studies identifying candidates for arthroscopic primary repair of the anterior cruciate ligament that patients older than 35 years and patients with a BMI under 26 had a higher chance of undergoing arthroscopic ACL primary repair, and that the operation within four weeks of injury was independently correlated with a higher likelihood of repair [24]. Looking into the outcomes between ACL repair with and without additional internal bracing, one of the studies revealed no statistical significant difference between failure rate or reoperation rate. No effect of age or time to surgery on clinical success was found either [25]. In a study comparing arthroscopic ACL primary repair with arthroscopic ACL reconstruction, no significant differences in knee stability could be detected between both groups with clinical examinations, Lachman and pivot-shift tests, or KT-1000 sideto-side measurements, after a mean follow-up of twenty-eight months [26]. A recent study investigating the outcomes of 11 patients who underwent arthroscopic primary ACL repair, it was found after two years, that the satisfaction and subjective scores were generally high, with the exception of one patient who failed because of noncompliance [27]. The same cohort of patients was followed for five years, one patient had a complex parrot-beak medial meniscus tear, following a twisting injury 2.5 years post-operatively, for which partial meniscectomy was performed. No deterioration (clinically relevant difference) was noted in any of the outcome scores between the two- and five-year outcomes [28].

The rehabilitation protocol differs as well; the main objectives were to control pain, swelling, and early range of motion. The differences observed were mainly on weight bearing status, the need for a splint or cast application and, range of motion allowed in the first four to six weeks, and time to commence patients on the standard ACL rehabilitation protocol.

The documented failure rates were mostly due to re-injury, non-compliance, or poorly selected patients for ACL repair.

The historical studies that looked at the outcome of ACL repair varied in sample size, gender difference, type of the ACL injury, surgical technique used, rehabilitation program implemented, follow-up duration, and the failure rate.
However, arthroscopic ACL repair can achieve a long-term clinical success in carefully-selected patients. The factors that can be inferred to favor a good-to-excellent clinical outcome in patients undergoing arthroscopic ACL primary repair are: acute ACL tear (proximal avulsion from its femoral attachment or close to it, young patients, absence of concomitant knee pathologies, good tissue quality of the ACL, and time to surgery within four weeks. The success is also driven by the advancement in the minimally invasive surgical techniques, experience of the operating surgeon, and patient's compliance.

Each individual study reviewed had few limitations, which are: 1) non-randomized retrospective studies, and hence, prone to selection bias, 2) small sample size, 3) variety of patients' age, 4) heterogeneous nature of cohort group, and surgical technique, 5) unknown cause of delay from injury to surgery, and 6) lack of objective assessment tool like KT1000 in some studies.

\section{Conclusion}

The current advancement in minimally invasive surgical techniques, fixation devices, pre-operative and post-operative rehabilitation program, arthroscopic primary ACL repair has profound advantages and can yield an excellent result in longer-term follow-up. However, appropriate patient selection is of paramount importance. Furthermore, a randomized control trial comparing the outcomes between arthroscopic primary ACL repair and reconstruction is highly recommended.

\section{Level of Evidence}

Level IV; Systematic Review.

\section{Source of Funding}

There were no external funding sources for this study.

\section{Conflicts of Interest}

The authors declare that they have no competing interests.

\section{References}

[1] Gluckert K, Kladny B, Blank-Schäl A, et al. MRI of the knee joint with a 3-D gradient echo sequence. Equivalent to diagnostic arthroscopy? Arch Orthop Trauma Surg 1992; 112: 5-14.

[2] Rupp S, Kaltenkirchen N, Hopf T, et al. Clinical relevance of tunnel position and interference screw location after replacement plasty of the anterior cruciate ligament with a patellar ligament transplant. Unfallchirurg 1995; 98: 650-54.

[3] Feagin JA Jr, Curl WW. Isolated tear of the anterior cruciate ligament: 5-year follow-up study. Am J Sports Med 1976; 4: 95-100. 
[4] Andersson C, Gillquist J. Treatment of acute isolated and combined ruptures of the anterior cruciate ligament. A longterm follow-up study. Am J Sports Med 1992; 20: 7-12.

[5] Andersson C, Odensten M, Good L, et al. Surgical or nonsurgical treatment of acute rupture of the anterior cruciate ligament. A randomized study with long-term follow-up. J Bone Joint Surg 1989; 71: 965-74.

[6] Engstrom B, Gornitzka J, Johansson C, et al. Knee function after anterior cruciate ligament ruptures treated conservatively. Int Orthop 1993; 17: 208-13.

[7] Radford WJ, Amis AA, Heatley FW. Immediate strength after suture of a torn anterior cruciate ligament. J Bone Joint Surg 1994; 76: 480-84.

[8] Odensten M, Lysholm J, Gillquist J. Suture of fresh ruptures of the anterior cruciate ligament. A 5-year follow-up. Acta Orthop Scand. 1984; 55: 270-72.

[9] Sommerlath K, Lysholm J, Gillquist J. The long-term course after treatment of acute anterior cruciate ligament ruptures. A 9 to 16 year follow up. Am J Sports Med. 1991; 19: 156-62.

[10] Fruensgaard S, Krøner K, Riis J. Suture of the torn anterior cruciate ligament. 5-year follow up of 60 cases using an instrumental stability test. Acta Orthop Scand. 1992; 63: 32325 .

[11] Trentacosta NE, Vitale MA, Ahmad CS. The effects of timing of pediatric knee ligament surgery on short-term academic performance in school-aged athletes. Am J Sports Med 2009; 37: 1684-91.

[12] Mastrangelo AN, Haus BM, Vavken P, et al. Immature animals have higher cellular density in the healing anterior cruciate ligament than adolescent or adult animals. J Orthop Res 2010; 28: $1100-6$.

[13] Mohtadi N, Grant J. Managing anterior cruciate ligament deficiency in the skeletally immature individual: a systematic review of the literature. Clin J Sport Med 2006; 16: 457-64.

[14] Daniel DM, Stone ML, Dobson BE, et al. Fate of the ACLinjured patient. A prospective outcome study. The American journal of sports medicine. 1994; 22: 632-44.

[15] Lohmander LS, Roos H. Knee ligament injury, surgery and osteoarthrosis: truth or consequences? Acta Orthop Scand 1994; 65: 605-9.

[16] Von Porat A, Roos EM, Roos H. High prevalence of osteoarthritis 14 years after an anterior cruciate ligament tear in male soccer players: a study of radiographic and patient relevant outcomes. Ann Rheum Dis 2004; 63: 269-73.

[17] Lohmander LS, Englund M, Dahl L, et al. The long-term consequence of anterior cruciate ligament and meniscus injuries. Am J Sports Med 2007; 35: 1756-69.

[18] Wester W, Canale ST, Dutkowsky JP, et al. Prediction of angular deformity and leg-length discrepancy after anterior cruciate ligament reconstruction in skeletally immature patients. J Pediatr Orthop 1994; 14: 516-21.

[19] Koman JD, Sanders JO. Valgus deformity after reconstruction of the anterior cruciate ligament in a skeletally immature patient. A case report. J Bone Joint Surg Am 1999; 81: 71115 .

[20] Streich NA, Barie A, Gotterbarm T, et al. Transphyseal reconstruction of the anterior cruciate ligament in prepubescent athletes. Knee Surg Sports Traumatol Arthrosc 2010; 18: 1481-86.

[21] Henry J, Chotel F, Chouteau J, et al. Rupture of the anterior cruciate ligament in children: early reconstruction with open physes or delayed reconstruction to skeletal maturity? Knee Surg Sports Traumatol Arthrosc 2009; 17: 748-55.

[22] Mastrangelo AN, Magarian EM, Palmer MP, et al. The effect of skeletal maturity on the regenerative function of intrinsic ACL cells. J Orthop Res 2010; 28: 644-51.

[23] Andersson C, Odensten M, Gillquist J. Early Arthroscopic Evaluation of Acute Repair of the Anterior Cruciate Ligament. Arthroscopy 1989; 5: 331-35.

[24] Van der List JP, Jonkergouw A, van Noort A, et al. Identifying candidates for arthroscopic primary repair of the anterior cruciate ligament: A case-control study. Knee 2019; 26: 61927.

[25] Jonkergouw A, van der List JP, DiFelice GS. Arthroscopic primary repair of proximal anterior cruciate ligament tears: outcomes of the first 56 consecutive patients and the role of additional internal bracing. Knee Surg Sports Traumatol Arthrosc 2019; 27: 21-28.

[26] Achtnich A, Herbst E, Forkel P, et al. Acute Proximal Anterior Cruciate Ligament Tears: Outcomes After Arthroscopic Suture Anchor Repair Versus Anatomic Single-Bundle Reconstruction. Arthroscopy 2016; 32: 2562-69.

[27] DiFelice GS, Villegas C, Taylor SA. Anterior cruciate ligament preservation: Early results of a novel arthroscopic technique for suture anchor primary anterior cruciate ligament repair. Arthroscopy 2015; 31: 2162-71.

[28] DiFelice GS, van der List JP. Clinical Outcomes of Arthroscopic Primary Repair of Proximal Anterior Cruciate Ligament Tears Are Maintained at Mid-term Follow-up. Arthroscopy 2018; 34: 1085-93.

[29] Hoffmann C, Friederichs J, von Rüden C, et all. Primary single suture anchor re fixation of anterior cruciate ligament proximal avulsion tears leads to good functional mid-term results: a preliminary study in 12 patients. J Orthop Surg Res 2017; $12: 171$.

[30] Fox J. M., Sherman O. H., Markolf K. Arthoscopic Anterior Cruciate Ligament Repair: Preliminary Results and Instrumented Testing for Anterior Stability. Arthroscopy 1985; 1: $175-81$. 